In conclusion, it is emphasized that the reduction procedure is always successful when applied to Ritz type difference equations; no claims are made about the relative merits of Ritz type difference equations compared to conventional equations in their accuracy in approximating the true solution.

Bettis Atomic Power Laboratory

Pittsburgh, Pennsylvania

1. L. Collatz, The Numerical Treatment of Differential Equations. Third Edition, SpringerVerlag, Berlin, Gottingen, Heidelberg 1960.

2. K. O. FriedrichS, A Finite Difference Scheme for the Neumann and the Dirichlet Problems. Report NYO-9760, Institute of Mathematical Sciences, New York University, 1962.

3. L. A. Hageman, Block Iterative Methods for Two-Cyclic Matrix Equations with Special Application to the Numerical Solution of the Second Order Self-Adjoint Elliptic Partial Differential Equation. Report WAPD-TM-327, Westinghouse Electric Corp., Bettis Atomic Power Laboratory, Pittsburgh 30, Pa., 1962. (Available from the Office of Technical Services, Dept. of Commerce, Washington 25, D. C.)

4. J. SchröDER, "Zur Lösung von Potentialaufgaben mit Hilfe des Differenzenverfahrens," Z. Angew. Math. Mech., v. 34, 1954, p. 241-253.

5. R. S. VARGA, Matrix Iterative Analysis, Prentice-Hall, Inc.; Englewood Cliffs, New Jersey, 1962.

\title{
A Two Parameter Test Matrix
}

\section{By P. J. Eberlein}

1. Introduction. The $(N+1) \times(N+1)$ matrix $A$ given by (1) below arose from a problem in the chemical theory of gases [1], the physically significant cases occurring when the parameter $s=0,1,2, \cdots$. Since the eigenvalues ${ }^{*}$ and eigenvectors of $A$ are found explicitly, the matrix is of interest in itself as a test matrix for eigenvalue programs, especially for negative real $s$ : when $s=-2,-3, \cdots-2 N$, the matrix is defective with two or more pairs of eigenvectors coalescing; elsewhere in the range $-2 N<s<-2$ at least one pair of eigenvectors is nearly parallel. In this range, the positive roots of the characteristic polynomial are ill-conditioned, especially for $s<-(N+1)$.

The matrix, its eigenvalues, and its right and left eigenvectors are given in section 2 ; a few numerical experiments are described in section 3 .

2. The Matrix. Let

(1) $\quad A=\left(a_{i j}\right)=\left(\begin{array}{ccccc}-N & N+s & 0 & 0 & \vdots \\ N & -(3 N+s-2) & 2(N+s-1) & 0 \\ 0 & 2(N-1) & -(5 N+2 s-8) & 3(N+s-2) & 0 \\ 0 & 0 & 3(N-2) & \cdot & \end{array}\right)$

Received February 1, 1963, revised November 10, 1963.

* Conjectured by Brauner and Wilson. 
or generally

$$
\begin{aligned}
a_{i i} & =-\left[(2 i+1) N+i s-2 i^{2}\right] \\
a_{i i+1} & =(i+1)(N+s-i) \\
a_{i i-1} & =i(N-i+1) \\
a_{i j} & =0 \quad|i-j|>1
\end{aligned}
$$$$
i, j=0,1,2 \cdots N
$$

$s$ is an arbitrary parameter, and $N+1$ is the order of the matrix.

The eigenvalues of $A$ are

$$
\lambda_{j}=-j(s+j+1) \quad j=0,1,2 \cdots N .
$$

Corresponding components of the left eigenvectors are

$$
x_{i}^{(j)}=\frac{1}{\left(\begin{array}{c}
N \\
i
\end{array}\right)} \sum_{k=0}^{q}(-1)^{k}\left(\begin{array}{c}
N-k \\
N-i
\end{array}\right)\left(\begin{array}{l}
j \\
k
\end{array}\right)\left(\begin{array}{c}
s+j+k \\
k
\end{array}\right), \quad i=0,1 \cdots N
$$

where $q=\min (i, j)$, or

$$
x_{N-m}^{(j)}=(-1)^{j} \frac{1}{\left(\begin{array}{l}
N \\
m !
\end{array}\right)} \sum_{p=0}^{r}(-1)^{p}\left(\begin{array}{l}
N-p \\
m-p
\end{array}\right)\left(\begin{array}{l}
j \\
p
\end{array}\right)\left(\begin{array}{c}
s+j+p \\
j
\end{array}\right)
$$

where $r=\min (m, j)$ and $m=N, N-1, \cdots 2,1,0$.

Let $n$ be an integer $1 \leqq n \leqq N$. The components of the right eigenvectors are

$$
y_{i}{ }^{(j)}=\left(\begin{array}{c}
N+s-i \\
N-i
\end{array}\right) x_{i}^{(j)} \quad \text { unless } s=-n \text { and } j \geqq n .
$$

If $s=-n$ and $j \geqq n$

$$
\begin{array}{rlrl}
y_{i}^{(j)^{\prime}} & =\left(\begin{array}{c}
N+s-i \\
N-i
\end{array}\right)^{\prime} x_{i}^{(j)} & & i \leqq N-n \\
y_{i}{ }^{(j)^{\prime}}=\left(\begin{array}{c}
N+s-i \\
N-i
\end{array}\right) x_{i}{ }^{(j)^{\prime}} & & i>N-n
\end{array}
$$

where the prime indicates that a factor $(n+s)$ has been deleted.

The proofs are left as an exercise for the reader at the suggestion of the referee. We note also that

If $s=2 n(1 \leqq n \leqq N)$ then $\lambda_{j}=\lambda_{2 n-j-1}$ and $x_{i}{ }^{(j)}=x_{i}{ }^{(2 n-j-1)}$ for $j=0,1$, $2 \cdots n-1$. If $s=-2 n-1(1 \leqq n \leqq N-1)$ then $\lambda_{j}=\lambda_{2 n-j}$ and $x_{i}{ }^{(j)}=x_{i}{ }^{(2 n-j)}$ for $j=0,1,2 \cdots n$.

3. Numerical Experiments. A few numerical experiments for various $N$ and $s$ have been made using the real program described in [2] on the IBM 7070 and the CDC 1604. The following examples were typical; the negative roots were found with good accuracy and the approximations to the positive roots were usually 
very bad. The non-defective cases, with several vectors nearly parallel, were sometimes worse than the defective cases.

$N=6 \quad s=-4$

$\begin{array}{llcllllll}\text { actual roots } & 0, & 0 & 2, & 2 & -1 & -4 & -10 & -18 \\ \text { approximations (7070) } & .0228 & -.0225 & 1.995, & 2.004 & -1 & -4 & -10 & -18\end{array}$

$N=6 \quad s=-6$

$\begin{array}{lllll}\text { actual roots } & -6 & 0,0 & 4,4 & 6,6 \\ \text { approximations }(7070) & -6 & -.0056 \pm .111 i & 3.969 \pm .363 i & 6.036 \pm .2215 i\end{array}$

$N=6 \quad s=-6.5$

$\begin{array}{lllllll}\text { actual roots } & -3 & 0 & 2.5 & 4.5 & 6 & 7,7.75\end{array}$

$\begin{array}{lllllll}\text { approximations (7070) } & -3 & .0066 & 2.436 & 5.103 & 5.151 & 7.401 \pm .405 i\end{array}$

The effect of higher precision is seen in the following example which was run on both the 7070 (8 digits) and the 1604 (10 digits)

$N=10 \quad s=-14$

actual roots approximations (CDC 1604) approximations (IBM 7070)

$0 \quad-2 \times 10^{-7} \quad .0026$

$12 \quad 12.00004 \quad 11.898$

$22 \quad 21.995 \quad 20.195$

$\begin{array}{lll}30,30 & 29.638 \pm 1.134 i & 23.843 \pm 7.16 i\end{array}$

$36,36 \quad 34.993 \pm 2.94 i \quad 33.926 \pm 12.92 i$

$40,40 \quad 40.024 \pm 3.39 i \quad 36.977$

$42,42 \quad 43.347 \pm 1.49 i \quad 46.387 \pm 10.99 i$

52.594

University of Rochester

Rochester, New York

1. J. Brauner \& D. J. Wilson, "Intramolecular reactions II: A weak energy transfer mechanism," J. Phys. Chem., v. 67, 1963, p. 1134-1138.

2. P. J. Eberlein, "A Jacobi-like method for the automatic computation of eigenvalues and eigenvectors of an arbitrary matrix," J. Soc. Indust. Appl. Math., v. 10, 1962, p. 74-88.

\section{Multivariate Polynomial Approximation for Equidistant Data}

\section{By B. Mond}

Abstract. The theory of polynomial approximation for evenly spaced points is extended to multivariate polynomial approximation. It is also shown how available tables prepared for univariate approximation can be used in the multivariate case.

1. Introduction. Assume $f(x)$ is given for $x=x_{1}, x_{2}, \cdots, x_{n}$ and it is desired to approximate $f(x)$ by a polynomial of degree $p, 1 \leqq p<n$, i.e.

$$
f(x) \approx \sum_{i=0}^{p} a_{i} x^{i}
$$

Received November 12, 1963. 